\title{
Single-nanosize pulverization of solid oxide by means of a wet planetary-bead-milling
}

\author{
Takaaki SAKAI, ${ }^{\dagger}$ Junji HYODO, ${ }^{*}$ Tatsumi ISHIHARA* and Hiroshige MATSUMOTO* \\ Environmental Technology Research Division, INAMORI Frontier Research Center, Kyushu University, \\ 744 Motooka, Nishi-ku, Fukuoka 819-0395, Japan \\ *Department of Applied Chemistry, Faculty of Engineering, Kyushu University, \\ 744 Motooka, Nishi-ku, Fukuoka 819-0395, Japan
}

\begin{abstract}
The single-nanosizing of a ceramics by a top-down method was demonstrated using a "wet planetary bead-milling" method, which is the combination of bead milling and planetary milling. By using the planetary bead-milling, single-nanoparticles (about $2 \mathrm{~nm})$ of $\mathrm{BaZr}_{0.9} \mathrm{Y}_{0.1} \mathrm{O}_{3-\alpha}(\mathrm{BZY}-91)$, which is one of the perovskite type oxides, was obtained with the combination of $0.05 \mathrm{~mm} \phi$ zirconia beads as pulverization media and ethanol as dispersion media.
\end{abstract}

(C2012 The Ceramic Society of Japan. All rights reserved.

Key-words : Single-nanosizing, Perovskite oxide, Planetary bead-milling, Top-down method, Proton conducting oxide

[Received October 4, 2011; Accepted October 6, 2011]

It has been widely known that various characteristics of materials change drastically from those of the bulk when nanosized. This is primarily attributed to the quantum size effect: Nanosizing leads to the decrease in the degeneracy of electronic band and its structure returns to discrete condition. In addition, the surface area increases, and thus the surface properties become pronounced. As a result, the various unique properties are developed in nanosized materials. For example, fluorescence characteristic of CdSe significantly changes in response to the reduction of the particle size. ${ }^{1-3)}$ Some non magnetic materials such as $\mathrm{Au}$ show ferromagnetic property when nanosized. ${ }^{4)}$ The sinterability of a material increases by the reduction of the particle size due to the increase in the surface free energy. ${ }^{5)}$ Superplasticity seen in a yttrium doped zirconia prepared through low temperature sintering of starting nano powder is another example of the benefit of ascendant surface properties. ${ }^{6), 7)}$ Since the unique properties become more pronounced as the particle size becomes smaller, methods to prepare nano materials, especially those for the preparation of "singlenanometer" materials, are extremely attractive; spherical 2-nmradius particles with typical density of metal oxide (e.g., $5 \mathrm{~g} \mathrm{~cm}^{-3}$ ) must have an extremely high specific surface area (higher than $10000 \mathrm{~m}^{2} \mathrm{~g}^{-1}$ ). However, the development of the preparation method for single-nano materials cannot be easily achieved.

There are two different approaches for nanosizing: One is based on the top-down methods that prepare nano-materials by breaking down a bulk material and the other is based on the bottom-up methods that prepare nano-materials by building up in atomistic scale. The methods that have been succeeded in preparing nano materials are mostly based on the latter; the typical methods are the coprecipitation method, ${ }^{8), 9)}$ the reverse micelle method, ${ }^{10)}$ the hot soap method, ${ }^{2)}$ the chemical vapor deposition (CVD) method, ${ }^{11)-13)}$ the sol-gel method, ${ }^{14)-17)}$ the salt

\footnotetext{
Corresponding author: T. Sakai; E-mail: sakai@ifrc.kyushu-u.ac.jp

* Corresponding author: H. Matsumoto; E-mail: matsumoto@ifrc. kyushu-u.ac.jp
}

assist spray pyrolysis (SASP) method, ${ }^{18), 19)}$ etc. Especially, the single-nano particle could be obtained in the hot soap method and reverse micelle method. ${ }^{2), 3), 10)}$

However, these bottom-up methods have restrictions in the choice of starting materials and/or preparation conditions. In addition, these methods have a difficulty in the application to complex compounds, e.g., metal oxides composing of different metals in controlled stoichiometry.

In contrast, the top-down method, i.e., pulverization, has an advantage in the point that the method is basically capable of wide application to various materials, even to the complex compounds, although this method has so far been fallen behind the bottom-up method in the achieved particle size. Therefore, the achievement of the "single-nanosizing" by top-down method will provide huge benefits.

Here we report that the single-nanosizing of a ceramics is enough possible by using a top-down method. The technique employed here is a wet planetary bead milling with submillimeter-diameter beads. A-few-nanometer particles of perovskite-type oxide could be obtained by using this technique.

Bead milling utilizes small milling ball, "beads" (the diameter of the beads is typically less than a few $\mathrm{mm}$ ), widely used not only for the pulverization, but also for mixing of inks and chemicals, in manufacturing paper, and etc. On the other hand, planetary-milling is one of the batch type milling methods, which pulverizes the sample by strong impact produced from planetary rotational motion, and can pulverize the sample more finely than the conventional milling using simple rotational motion.

These methods themselves are not quite unique. However, combination of these methods provides us much fine pulverization. We reported previously the reduction of sintering temperature of a ceramic, a perovskite series oxide of $\mathrm{BaZr}_{0.9} \mathrm{Y}_{0.1} \mathrm{O}_{3-\alpha}$, by preparing the green slurry using this method. ${ }^{5)}$ In the present report, we show our finding that the solid oxide can be pulverized into single nanosize particles: about " $2 \mathrm{~nm}$ " in most frequent diameter, which is extremely difficult level in the usual ceramics pulverization. The resultant sample was characterized by powder $\mathrm{X}$-ray diffraction (XRD), small angle X-ray scattering (SAXS), 


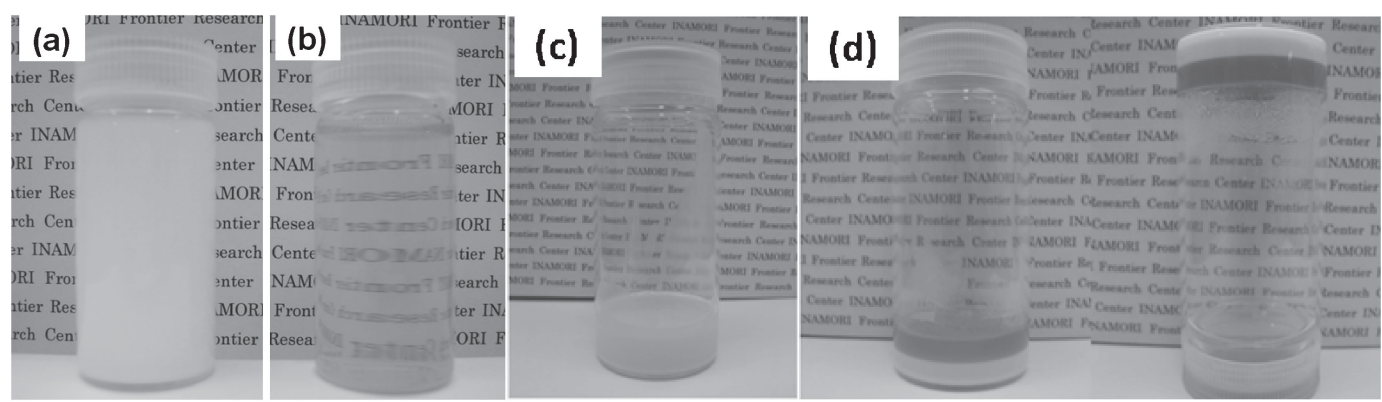

Fig. 1. Photograph of the $2 \mathrm{wt} \%$ slurries after milling at $500 \mathrm{rpm}$ for $1 \mathrm{~h}$ with 2 -mm $\phi$ beads (a) and after further milling at $800 \mathrm{rpm}$ for $7 \mathrm{~h}$ with $0.05-\mathrm{mm} \phi$ beads (b) contained in $10-\mathrm{mL}$ glass bottles, and Photographs of the $20 \mathrm{wt} \%$ slurry after further milling at $800 \mathrm{rpm}$ for $7 \mathrm{~h}$ with 0.05 -mm $\phi$ beads; the photographs right after the milling (c) and a few days after the milling (d). The white part of the bottom of the slurry was $0.05-\mathrm{mm} \phi$ beads.
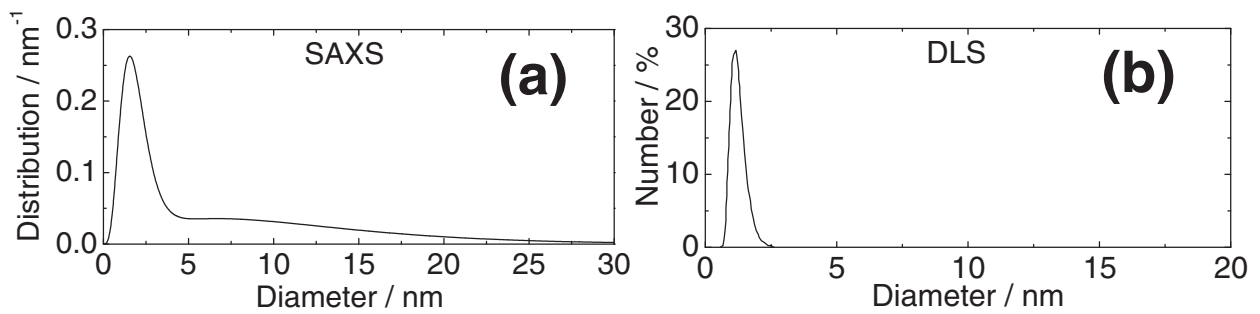

Fig. 2. The particle diameter distribution of the BZY-91 particles milled at 0.05 -mm $\phi$ measured by small angle X-ray scattering (SAXS) (Ultima IV, Rigaku corp.) (a) and dynamic light scattering (DLS) (Zetasizer nano series, Sysmex corp.) (b). In the case of DLS, the slurry was treated in an ultracentrifuge at about $77,000 \mathrm{G}$ for $1 \mathrm{~h}$ to remove larger particles since the smaller particles cannot be observed in the DLS when the larger particles exist. Therefore, the number of the particles over $3 \mathrm{~nm}$ was reduced in the DLS data.

transmission electron microscopy (TEM), and dynamic light scattering (DLS) to prove how actually the oxide nanoparticles were obtained by this top-down sort of method with brief report of methodology.

$\mathrm{BaZr}_{0.9} \mathrm{Y}_{0.1} \mathrm{O}_{3-\alpha}$ (BZY-91) powders were prepared by a solidstate reaction method. $\mathrm{BaCO}_{3}, \mathrm{ZrO}_{2}$, and $\mathrm{Y}_{2} \mathrm{O}_{3}$ powder $(99.9 \%$ pure) were wet-blended using ethanol and then calcined at $1250^{\circ} \mathrm{C}$ for $10 \mathrm{~h}$ in air. The BZY-91 powders obtained were confirmed as single phase by XRD measurement.

The zirconia beads of 2 and $0.05 \mathrm{~mm} \phi$ were used for the milling and ethanol was used as a dispersion medium. Sample powders of BZY-91, ethanol and 2-mm $\phi$ zirconia beads were put into zirconia pot of $45 \mathrm{~mL}$, and were planetary-milled at $500 \mathrm{rpm}$ for $1 \mathrm{~h}$ by using a planetary ball mill (P-7 classic, Fritsch Co., Ltd.), thereby affording the white slurry. The slurry was separated by a sieve from the zirconia beads, and was put into the zirconia pot again with $0.05-\mathrm{mm} \phi$ zirconia beads, and was planetarymilled at $800 \mathrm{rpm}$ for $7 \mathrm{~h}$. The amounts per pot of the sample powder, the dispersion medium and beads were 1,19 and $50 \mathrm{~g}$, respectively.

Figure 1 shows photos of the slurries after milling with 2-mm $\phi$ beads [Fig. 1(a)] and further milling with $0.05-\mathrm{mm} \phi$ beads [Fig. 1(b)]. The amounts of the oxide sample in the slurries were almost the same (about $2 \mathrm{wt} \%$ ) in both slurries. After milling with $2-\mathrm{mm} \phi$ beads, the slurry was at first opaque [Fig. 1(a)], and separated into precipitates and ethanol in a short time. In contrast, after milling with $0.05-\mathrm{mm} \phi$ beads, the slurry became semitransparent and did not form precipitation. Such an appearance of the milled sample suggests a strong possibility that the particle size became significantly small by planetary bead milling with 0.05 - $\mathrm{mm} \phi$ beads.
These features depend on the choice of dispersion medium. The semi-transparent slurry could not be obtained by using other dispersion media such as water, isopropanol, cyclohexane, ethylene glycol; ethanol is the only successful dispersion medium for BZY-91 to obtain transparency of the slurry within the present examination. On the other hand, the ratio of the sample to ethanol and beads ( 1 to 19 and $50 \mathrm{~g}$, respectively) in the present condition is fairly small. Further increase in the sample weight results in gelation of the slurry and hence interfere complete milling. In addition, the gelation makes the separation of the slurry from beads difficult; the separation became almost impossible when the fraction of the oxide sample was $20 \mathrm{wt} \%$. Figure 1 also shows the photos of $20 \mathrm{wt} \%$ slurries with $0.05 \mathrm{~mm} \phi$ beads right after milling [Fig. 1(c)] and a few days after the milling [Fig. 1(d)]. The slurry right after the milling is highly viscous and is hardly separated from $0.05 \mathrm{~mm} \phi$ beads by the sieve. In addition, as shown in Fig. 1(d), the slurry easily solidifies within a few days under standing-still condition.

Evidently, the pulverization of the BZY-91 oxide powder to the semi-transparent state owes to a proper choice of the dispersion medium and the sample weight against beads and dispersion medium.

The particle size of the obtained semi-transparent slurry (0.05$\mathrm{mm} \phi$ bead-milled sample) was evaluated by small angle X-ray scattering (SAXS) and dynamic light scattering (DLS). As shown in Fig. 2, the particle size was almost in single-nanometer scale with the most frequent diameter of about " $2 \mathrm{~nm}$ " in both of the measurements. These two measurements, SAXS and DLS, utilize different principles to generate the particle size distribution, and thus the similar results derived from the two technique suggest 


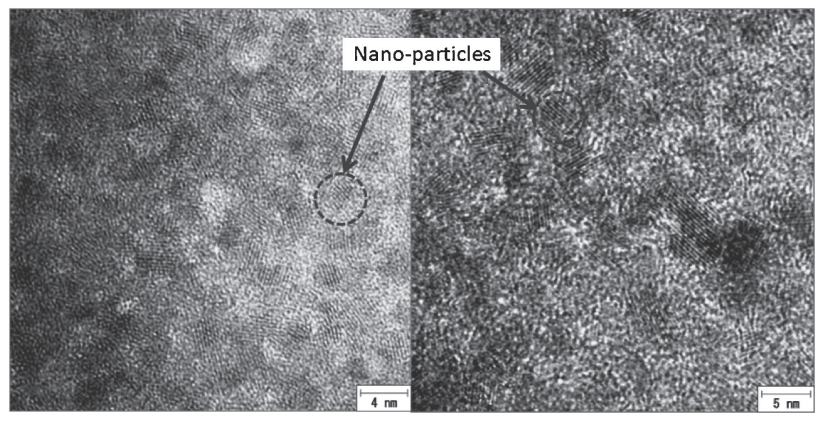

Fig. 3. Images of the BZY-91 nano particles bead-milled at $0.05-\mathrm{mm} \phi$ measured by transmission electron microscope (EM-002B, TOPCON corp.). The measurement was performed up to 590,000-fold magnification with $200 \mathrm{kV}$ of acceleration voltage.

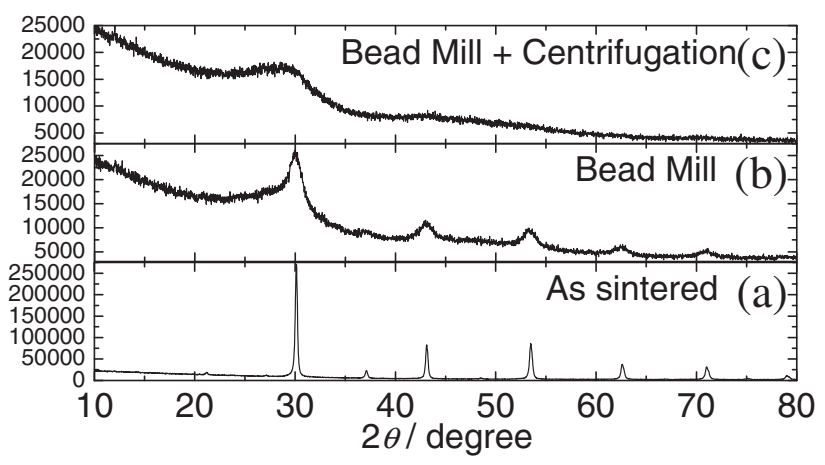

Fig. 4. XRD pattern of as-sintered BZY-91 (a), bead-milled BZY-91 (b) and bead-milled BZY-91 with ultracentrifugation (c) measured by powder X-ray diffraction (Ultima IV, Rigaku Corp.). The ultracentrifugation was also performed at about $77,000 \mathrm{G}$ for $1 \mathrm{~h}$, i.e., the pattern of the ultracentrifugation sample was that of single-nanometer particles as shown in DLS data [Fig. 2(b)]

that the milled sample are actually composed of single-nanometer particles.

Figure 3 shows $0.05-\mathrm{mm} \phi$ bead-milled sample directly observed by transmission electron microscopy (TEM). Numerous particles distinguished with others and surroundings by lattice pattern can be seen in the pictures with the size in single nanoscale, i.e., smaller than the scale bar (4 and $5 \mathrm{~nm}$ ). This microscopic observation assures us that single nanoparticles were the main component of the sample processed by the planetary-bead-milling with $0.05-\mathrm{mm} \phi$ beads. The lattice plane spacing of the lattice images observed in Fig. 3 was about $0.3 \mathrm{~nm}$, which corresponds to the lattice spacing of $\langle 110\rangle$ plane of BZY-91. This result suggests that the BZY-91 powders were pulverized into significant small cluster without losing the original crystal structure.

The XRD pattern of the particles is shown in Fig. 4. It can be seen in Fig. 4(b) that the XRD pattern of the slurry after the bead milling becomes broad compared with that of the powder before milling [Fig. 4(a)], also suggesting BZY-91 powder was finely pulverized. Figure 4(c) illustrates the XRD pattern of the beadmilled slurry after ultracentrifugal separation at $77,000 \mathrm{G}$ for $1 \mathrm{~h}$. In this case, relatively large particles were removed by the ultracentrifugation.

The XRD pattern in Fig. 4(c) became so broad as to be indistinguishable clearly. However, as compared with Figs. 4(a) and 4(b), it can be seen that the smooth peaks exist at the positions at which the diffraction peaks are observed in Figs. 4(a) and 4(b), indicating that the milled specimen of BZY-91 keeps the original structure. Evidently, the singlenanoparticles are not the product of decomposition or chemical reaction, but come from the original perovskite-type oxide. The planetary bead milling provides the crashing of the ceramic specimen into nanoparticles, so that the original crystal structure is maintained.

Meanwhile, a concern lies in the contamination, which is attributed to the high ratio of beads weight against sample weight as well as high speed of planetary rotation. This time, the discussion of the contamination is difficult since zirconium, which is main component of bead, is also contained in the sample. Further work is necessary to discuss the contamination from beads.

Finally, the advantage of this planetary-bead-milling is that this method is applicable for various materials. This method has a high potential since there is a possibility that various single nano-materials is obtained by applying this method to other materials.

As mentioned in introduction, there is strong demand for fine pulverization of functional materials since its nanosizing will provide a huge benefit. Top-down method is suitable for fulfillment of the demand because top-down method does not need to prepare any complexed procedure for nanosizing of respective material and just simply pulverizes a bulk material. This simpleness is significantly useful from the perspective of industry as well as science. The main point of this paper is that single-nanosizing can be possible by using top-down method. The planetary bead milling is one of the ways for the singlenanosizing by the top-down method, and the single-nanosizing by top-down method might be achieved by various milling methods other than the planetary bead milling.

Acknowledgement This study was supported by Grant-in-Aid for Scientific Research (B) (21360481), from Japan Society of the Promotion of Science (JSPS).

\section{References}

1) C. B. Murray, D. J. Norris and M. G. Bawendi, J. Am. Chem. Soc., 115, 8706-8715 (1993).

2) H. Nakamura, Y. Yamaguchi, M. Miyazaki, H. Maeda, M. Uehara and P. Mulvaney, Chem. Commun. (Camb.), 28442845 (2002).

3) G. Schmid, "Nanoparticles-From Theory to Application", Willey-VCH $\mathrm{GmbH}$ and $\mathrm{Co}, \mathrm{KGaA}$, Weinheim, Germany (2004) pp. 48-76.

4) Y. Yamamoto, T. Miura, M. Suzuki, N. Kawamura, H. Miyagawa, T. Nakamura, T. Teranishi and H. Hori, Phys. Rev. Lett., 93, 116801 (2004).

5) T. Sakai, H. Matsumoto, Y. Sato, J. Hyodo, N. Ito, S.-i. Hashimoto and T. Ishihara, Electrochemistry, 77, 876-878 (2009).

6) F. Wakai, S. Sakaguchi and Y. Matsuo, Adv. Ceram. Mater., 1, 259-263 (1986).

7) K. Kajihara, Y. Yoshizawa and T. Sakuma, Acta Metall. Mater., 43, 1235-1242 (1995).

8) S. Rajendran and H. J. Rossell, J. Mater. Sci., 26, 5815-5822 (1991).

9) K. Kaneko, H. Ihara, M. Hirabayashi, N. Terada and K. Senzaki, Jpn. J. Appl. Phys., 26, L734-L735 (1987).

10) M. L. Steigerwald, A. P. Alivisatos, J. M. Gibson, T. D. Harris, R. Kortan, A. J. Muller, A. M. Thayer, T. M. Duncan, D. C. Douglass and L. E. Brus, J. Am. Chem. Soc., 110, 3046-3050 (1998). 
11) K. Wegner, W. J. Stark and S. E. Pratsinis, Mater. Lett., 55, 318-321 (2002).

12) Y. Azuma, M. Shimada and K. Okuyama, Chem. Vap. Deposition, 10, 11-13 (2004).

13) T. Seto, M. Shimada and K. Okuyama, Aerosol Sci. Technol., 23, 183-200 (1995).

14) A. Pohl, G. Westin and K. Jansson, J. Sol-Gel Sci. Technol., 26, 257-260 (2003).

15) C. Miranda, M. E. V. Costa, M. Avdeev, N. A. L. Kholiki and
J. L. Baptista, J. Eur. Ceram. Soc., 21, 1303-1306 (2001).

16) C. Sanchez and J. Livage, N. J. Chem., 14, 513-521 (1990).

17) T. Sakai, S. Kajitani, S.-J. Kim, J. Hamagami, H. Oda, M. Matsuka, H. Matsumoto and T. Ishihara, Solid State Ionics, 181, 1746-1749 (2010).

18) B. Xia, I. W. Lenggoro and K. Okuyama, Adv. Mater., 13, 1579-1582 (2001).

19) B. Xia, I. W. Lenggoro and K. Okuyama, J. Mater. Chem., 11, 2925-2927 (2001). 